\title{
Anderson-Fabry disease: a histopathological study of three cases with observations on the mechanism of production of pain
}

\author{
PAULINE KAHN \\ From the Pathology Department, Maida Vale Hospital, London and the Department \\ of Neuropathology, the Institute of Psychiatry and the Maudsley Hospital, De Crespigny Park, London
}

SUMMARY A clinical review and histopathological study of three cases of Anderson-Fabry disease is presented and pathological changes in the central and peripheral nervous systems are reported, in some sites for the first time. These are telangiectatic changes in vessels of the sympathetic ganglia in the vertebral trunk; storage of glycolipid in pigmented cells of the substantia nigra and in anterior horn cells; and degeneration of nerve fibres in the dorsal root entry zone and substantia gelatinosa of the spinal cord. The histopathological findings suggest that in this disease pain is due to involvement of dorsal root ganglion cells with associated axonal degeneration of the small fibres in pathways subserving pain.

Anderson-Fabry disease (AFD), also known as angiokeratoma corporis diffusum or Fabry's disease, is a rare inborn error of glycosphingolipid metabolism and belongs to the group of sphingolipidoses (Sweeley and Klionsky, 1963). The enzyme ceramide trihexosidase is deficient or absent, with the result that there is accumulation of abnormal glycolipid, predominantly ceramide trihexoside, in various organs (Brady et al., 1967).

The earliest descriptions of the disease were by Anderson (1898) in England and Fabry (1898) in Germany. For the next 50 years several cases were recognized and were reported in journals of dermatology, since a skin rash is often one of the early manifestations. Pompen and Ruiter (1947) were the first to suggest that the disease might be a generalized storage disorder. Sweeley and Klionsky $(1963,1966)$ isolated from organs of patients with Anderson-Fabry disease two glycolipids which they identified as ceramide dihexoside and ceramide trihexoside, confirming Pompen and Ruiter's suggestion.

The metabolic defect is transmitted by a sexlinked gene with constant penetrance in the homozygous male and occasional penetrance in

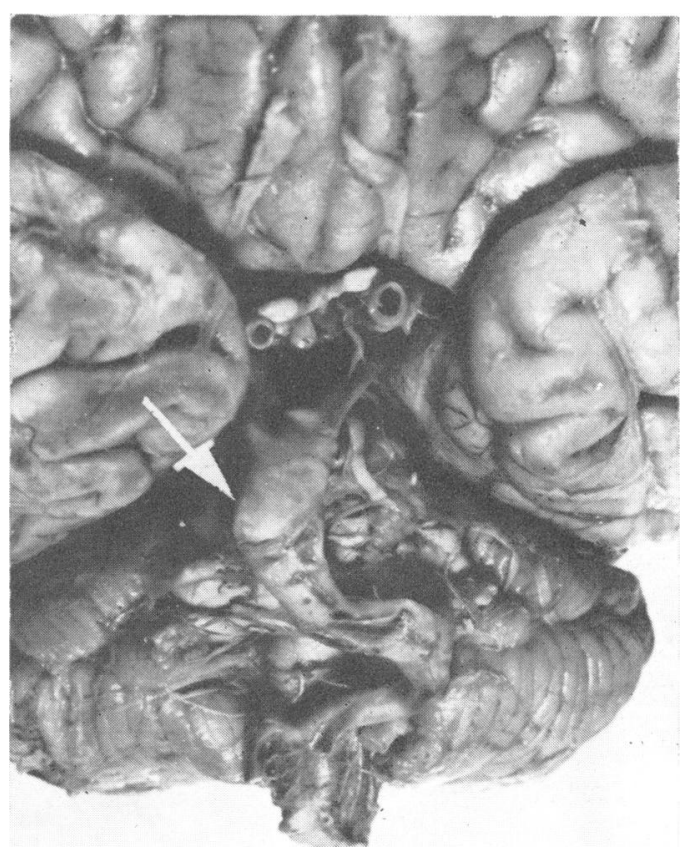

FIG. 1. Brain-stem. The basilar artery is dilated and tortuous. There is a flat well-defined 'plaque' at the rostral end (arrow). 
the heterozygous female (Wise et al., 1962; Opitz et al., 1965).

In males, Anderson-Fabry disease becomes manifest in childhood or early adolescence with intermittent and often excruciating pain in the fingers and toes, the characteristic skin eruption at this stage often being absent or ill-developed. Sweating and oedema may also precede the skin eruption by many years (Wallace, 1973). The characteristic eruption consists of superficial telangiectatic, dark red, sometimes almost black macules and papules, usually up to $2 \mathrm{~mm}$ in diameter. These are found over the trunk and limbs, most densely clustered over the lower trunk and thighs. The eruption is seldom conspicuous before the late teens and tends to become more profuse during the third and fourth decades.

The pain occurs in $90 \%$ of males and in less than $10 \%$ of females, attacks usually beginning between the ages of 5 and 15 years (Wallace, 1973). The site of the pain bears no relation to the site of the rash and occurs mainly in the fingers and toes, sometimes spreading proximally. The pain has a peculiar burning character, a combination of constant heat and aching felt deep to the skin, and difficult for the patient to describe. There are also incapacitating exacerbations of a lancinating pain which may be brief or last up to three weeks. These occur especially in childhood when there is sometimes an accompanying pyrexia. Extremes of temperature, exertion, and vibration may provoke or aggravate the pain. Certain movements can cause exacerbations; a patient may suddenly stop and cry out with pain provoked by raising a spoon to his mouth. He may be very distressed during such an episode and present a bizarre picture. Affected children may be labelled neurotic and many medical opinions may be sought before the condition is correctly diagnosed. There is sometimes a strong emotional component and adults may be reduced to tears by their suffering. Not all patients experience the same degree of pain. In some cases there are periods of complete remission. Local vasodilatation may occur during an attack and less frequently, vasoconstriction. Cutaneous sensation is normal.

Small corneal opacities are found in the fully developed cases and in asymptomatic hetero zygotes. These faint grey streaks or blotches mayo be detected at a very early age and have been reported in a boy aged 6 months (Spaeth and Frost, 1965) and in a girl aged 3 years (Colombi et al., 1967). This corneal dystrophy is mimickedo only by chloroquin keratopathy and constitute

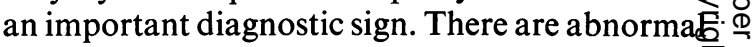
dilated and tortuous conjunctival and retina $\vec{F} \vec{\omega}$

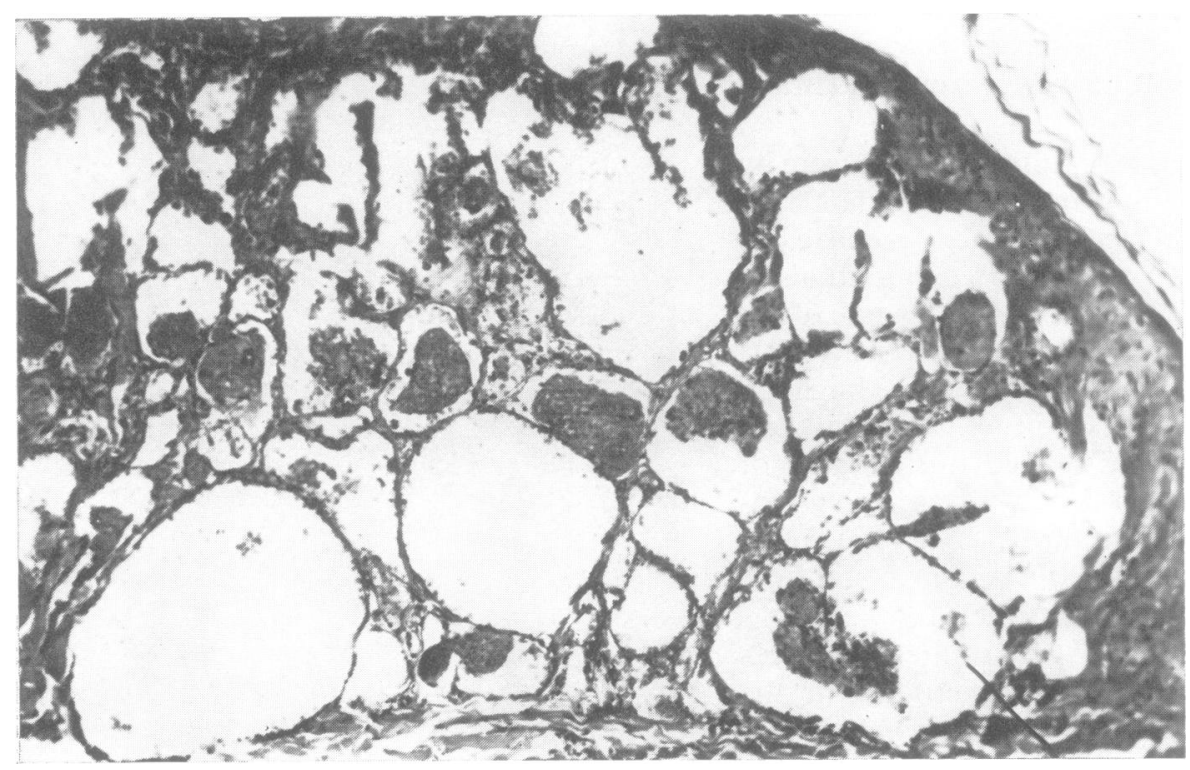

FIG. 2. Skin: telangiectatic vascular spaces in the upper dermis and atrophy of the overlying dermis. $H$ and $E, \times 200$. 


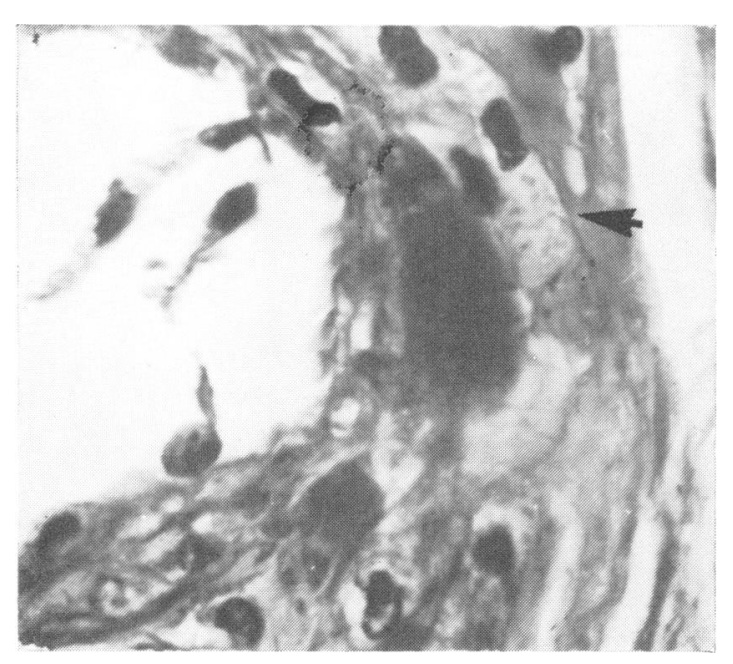

FIG. 3. A small leptomeningeal artery shows thickening and deposits of glycolipid in the media. There is also a macrophage with foamy cytoplasm (arrow). Luxol fast blue, $\times 770$.

vessels. The abnormal retinal vessels are seen early in life and are not related to hypertension.

Proteinuria, anaemia, colitis, cardiovascular disorders, and disturbances of the nervous system follow in the ensuing years. Minor cerebrovascular episodes are common in the form of small strokes and the brain-stem is particularly vulnerable. These episodes occur in young adults when the blood pressure is normal. Symptoms of autonomic imbalance and diabetes insipidus are encountered. Death may occur in severe cases in early adult life, usually due to uraemia. Other common causes of death are myocardial infarction and cerebral haemorrhage. The average expectation of life in males is 42 years. No male patient is known to have survived beyond the age of 64 years.

The characteristic histopathological findings in Anderson-Fabry disease are the telangiectatic lesions in the upper dermal layer of the skin and glycolipid deposits in various sites. Glycolipid is deposited in capillary endothelium, in the smooth muscle of blood vessels throughout the body, in myocardium, in the kidney, uterus, lymph nodes, cells of the reticuloendothelial system, and in selected sites in the nervous system. The glycolipid is faintly eosinophile and stains deeply with

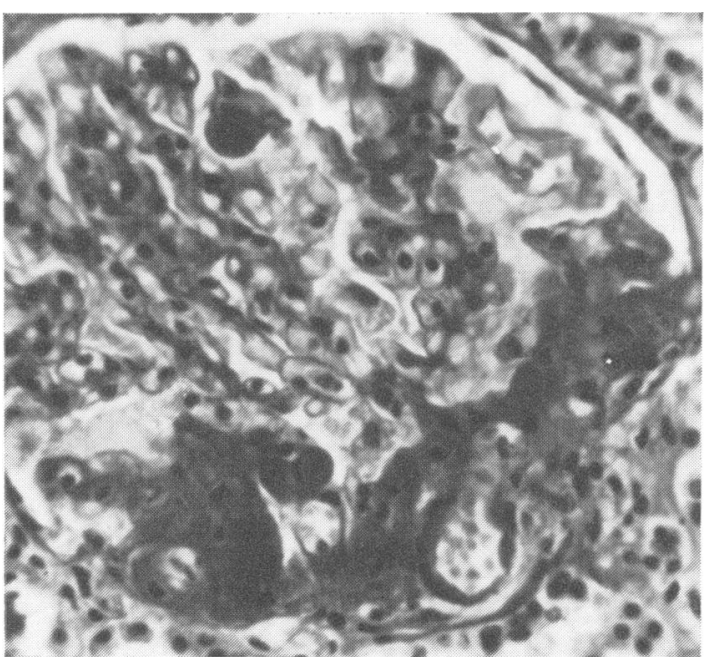

FIG. 4. Kidney. There is a mass of dense hyaline material in the glomerulus near the afferent arteriole and pale foam cells in the epithelium of the glomerular tuft. $H$ and $E, \times 350$.

periodic acid Schiff, Luxol fast blue, Oil red O, and Sudan black B.

\section{PRESENT STUDY}

The purpose of the present study is to present the general and neuropathological findings in case 1 (G.W.); to summarize and review the neuropathological findings in case 2 (H.W.), the brother of case 1 ; and to present the neuropathological findings in case 3 , the grandson of Anderson's original case of 'angeio-keratoma'.

\section{CASE 1}

Details of the early clinical history of this patient are documented in the reports of Wise et al. (1962) and of Steward and Hitchcock (1968). Briefly, the patient was born in 1937 and developed symptoms at the age of 7 years. A rash on the trunk and thighs was followed by the onset of severe burning pain in the hands, feet, and genitals. Episodes of severe pain occurred in association with fever. Pethidine was the only drug which alleviated his pain. In the ensuing years he developed albuminuria, anaemia, colitis, polymyositis, and suffered six cerebrovascular accidents, the first at the age of 21 years. In 1965, at the age of 28 years, he was admitted to hospital as he could no longer walk. There was spastic paraparesis, 


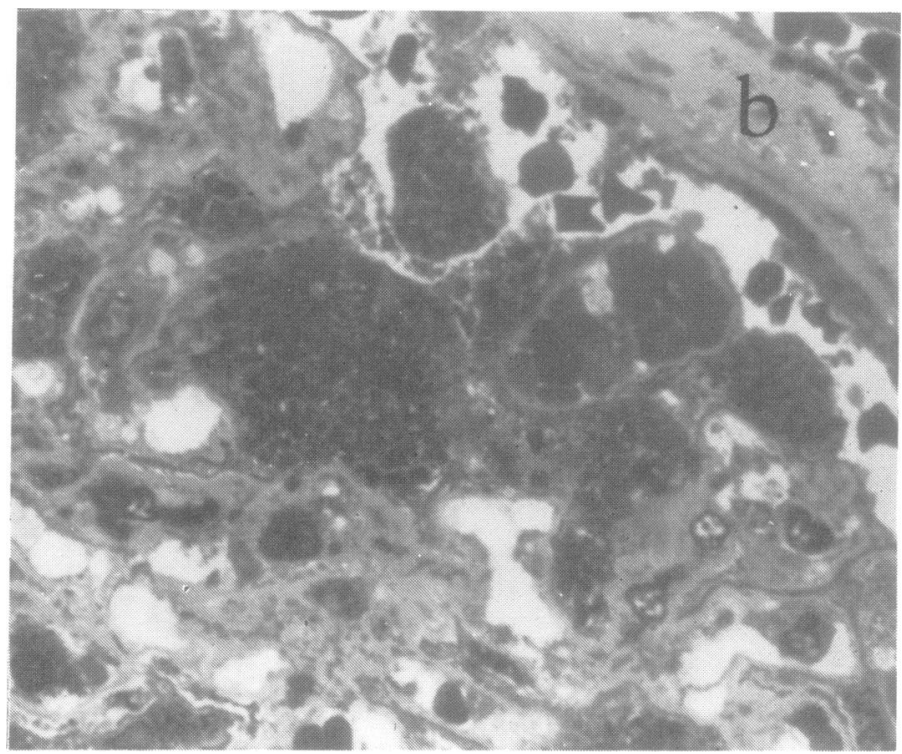

FIG. 5. Glomerulus of the kidney. Granules of glycolipid in the cytoplasm of podocytes. The basement membrane (b) is grossly thickened. Toluidine blue, $\times 600$.

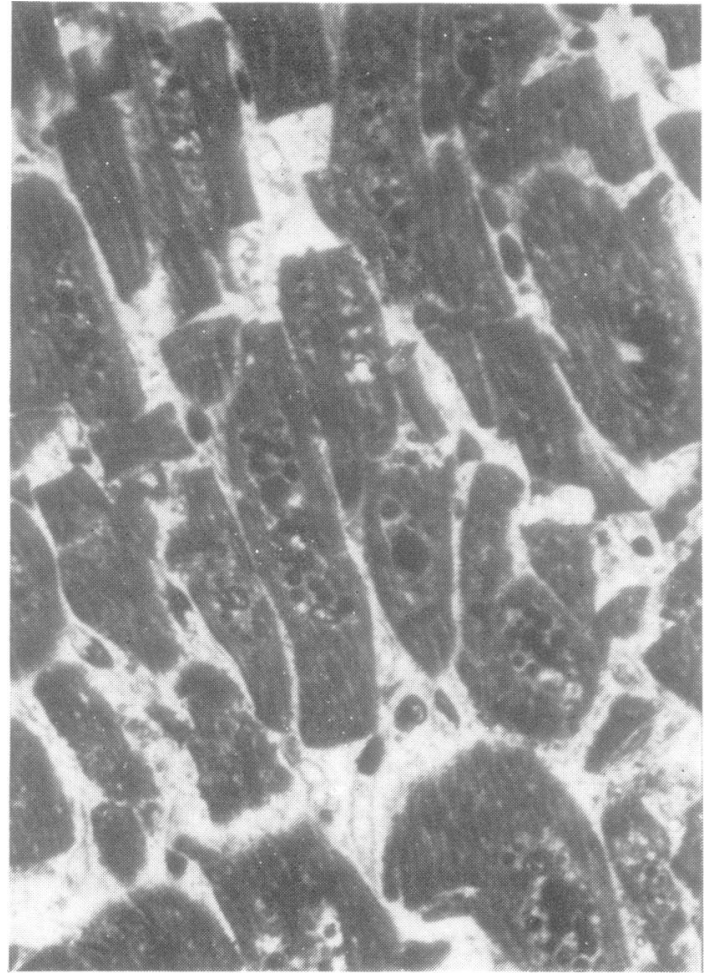

FIG. 6. Myocardium. Vacuoles in the enlarged muscle fibres contain coarse lipid droplets. Toluidine blue, $\times 600$. more severe on the right side, bilateral ptosis, dilated sluggish left pupil, and weakness of the righto trapezius muscle. He was dysarthric. The cerebro $\Omega_{\mathbb{2}}-$ spinal fluid was clear, and colourless, with a proteino of $85 \mathrm{mg} / 100 \mathrm{ml}$. The Lange curve was 000111 . A diagnosis of left anterior cerebral artery thrombosiso was made. The patient was never hypertensive. Hes. and his brother (case 2) were nursed at home by theifo mother. He received large doses of pethidine and chlorpromazine. Death occurred in 1968 at the age of 31 years of renal failure.

The patient's mother had no symptoms of the disease but an inconspicuous rash and corneal opacities were found on examination for signs of Anderson-Fabry disease. There was a third son in the family who was free of the disorder. The mother had five brothers and two sisters, all of whom were unaffected, and had healthy children.

At postmortem examination the body was that of a cachectic young man with contractures of all limbs and widespread dark red macular and papular lesions up to a few millimetres in diameter over the trunk, thighs, forearms, and mucosa of the lips, which were prominent. The skin of the ankles and feet showed oedema and induration resembling chronic lymphoedema. There was generalized muscular wasting, very severe in the arms and hands. The heart weighed $315 \mathrm{~g}$. The myocardium was extremely pale. There was no atheroma of the aorta or the vessels in the neck. Thyroid, pancreas, and kidneys were remarkable for their extreme pallorthe colour of putty. The kidneys (right $90 \mathrm{~g}$, left $115 \mathrm{~g})$ showed scarring and prominent vascular 


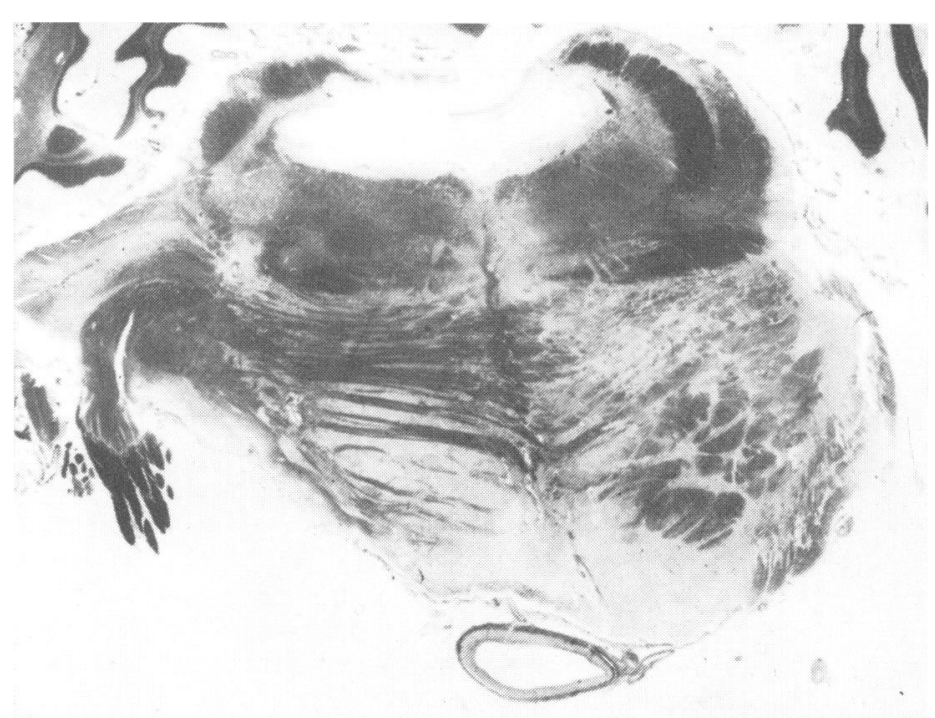

FIG. 7. Pons. There is shrinkage of the anterolateral pons. The basilar artery is dilated and thin walled. Haematoxylin-van Giesen, $\times 2$.

marking of the capsular surfaces. The cut surfaces showed a pale narrow irregular cortex with vascular markings and a very pale medulla.

The brain $(1,220 \mathrm{~g})$ was atrophic and extremely pale. The pons was flattened on the left anterolateral aspect. Coronal sections of the brain showed moderate ventricular dilatation. There were several small cerebral and cerebellar cortical infarcts. There was no infarct in the anterior cerebral artery territory. In the pons there was a large, partly cystic infarct in the left basis pontis which extended across the midline to involve the medial portion of the right side. The left pyramid in the medulla was shrunken and grey, resulting from the massive infarction in the pons.

\section{CASE 2}

Details of the clinical history are documented in the reports of Wise et al. (1962) and of Steward and Hitchcock (1968). The patient was born in 1933. At the age of 9 years he first felt pain in his toes when playing football and soon the pain began to involve his fingers. A symmetrical rash on his body and limbs, densely clustered in the 'bathing trunk' area was noticed at the age of 10 years. He was free of pain for periods, but developed episodes of fever with pain in the fingers and toes. He became subject to more or less continuous pain for which he received large doses of pethidine in later years. He developed ophthalmic herpes and said that the pain was mild compared with the pain in his extremities. At the age of 29 years he had intermittent weakness of the leg; then he developed myoclonic jerking of the legs. This jerking became violent so that he fractured the neck of the right femur. The hip became infected after operation and he died three weeks later from pulmonary embolism.

The postmortem findings of Dr. J. Reed were reviewed and reported by Steward and Hitchcock (1968). There were characteristic skin lesions and lipid deposits in smooth muscle, kidneys, connective tissue, and in the nervous system. The hypothalamus, spinal cord, dorsal root ganglia, and cauda equina were processed and examined in the course of the present study.

Chemical analysis of portions of the brains and kidneys in cases 1 and 2 was undertaken by Professor J. N. Cumings (1971). No abnormality was detected in the frontal cortex or white matter in either case. There was a striking increase in ceramide dihexoside and ceramide trihexoside in the kidneys. The plasma lipids were normal.

CASE 3

The patient was born in 1921. At the age of 15 years he developed ulceration of both lower legs after a football injury. He had meningitis soon after and then developed the characteristic eruption of angiokeratoma particularly over the lower trunk and thighs, slight peripheral pain, and heat intolerance. At the age of 41 years he attended St. Thomas's Hospital, London, where he was under the care of Dr. H. J. Wallace. Anderson-Fabry disease was diagnosed by examination of the skin and by renal biopsy. There was corneal dystrophy, anaemia, and 


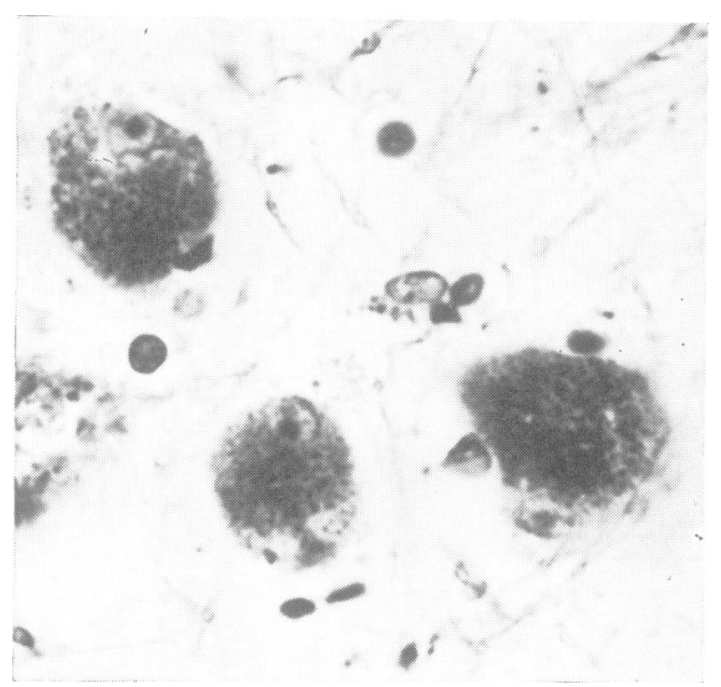

FIG. 8. Amygdala. A group of neurones distended by fine lipid granules. Luxol fast blue, $\times 770$.

proteinuria. Renal function tests were normal until the last two years of life. He remained at work, though latterly of a very light nature, until six months before his death. During this period he was twice admitted to hospital for epileptiform convulsions. At this time there was gross impairment of renal function and renal biopsy showed very severe atrophic changes in the kidney. The patient developed

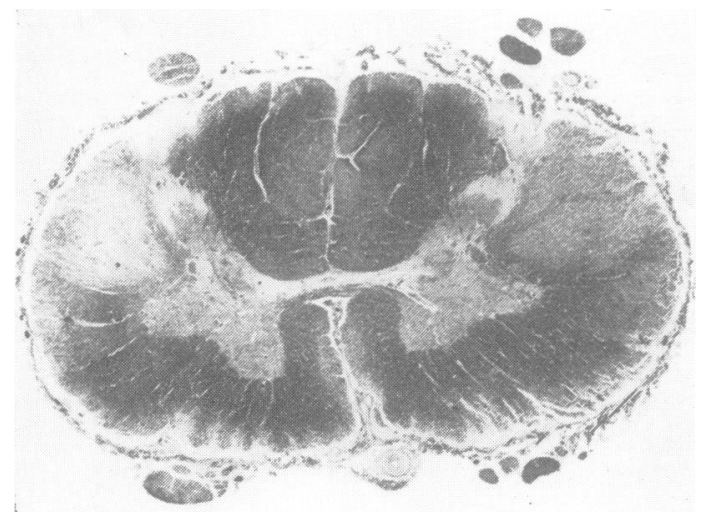

FIG. 9. Spinal cord. There is pallor of the corticospinal tracts on myelin stained sections. The dorsal columns are normal. Heidenhain's iron haematoxylin, $\times 7$. signs of cerebral ischaemia. He died in 1971 at the age of 50 years.

The patient's mother was the first recorded instance of Anderson-Fabry disease in the female, the diagnosis being made by retrospective examination of necropsy material in brain and kidney (Wallace, 1958). One male maternal cousin also died with this disorder at the age of 39 years, with renal failure and hypertension.

At postmortem examination (Dr. J. Tighe) the body was that of a well-nourished male. There was gross oedema with thickening and warty excrescences of the skin of the feet and lower half of both legs below the knee: the appearance of chronic lymphoedema. Small pin-head angiomata were present on the lower trunk, buttocks and thighs. The heart weighed $605 \mathrm{~g}$. There was no gross atheromatous change in the aorta or arteries in the neck. Both kidneys were small with pronounced vascular markings and focal areas of lipid deposition on the capsular surfaces, and reduction of renal parenchyma with loss of demarcation of cortex and medulla on cut surfaces.

The brain weighed $1390 \mathrm{~g}$ and showed thickeningo of the pia-arachnoid membranes, most marked at the base and over the frontal convexities. The vertebral and basilar arteries showed massive dilata tion. The basilar artery was elongated and tortuous and there were lipid plaques resembling atheromatous plaques in its wall (Fig. 1). Coronal sections of the brain showed moderate symmetrical ventriculaf dilatation. The aqueduct of the midbrain was dilated? The substantia nigra was narrow but well pigmented. Cerebellum and spinal cord were of normal appearance.

METHODS FOR HISTOLOGICAL STUDY OF TISSUES Frozen, paraffin-wax, and celloidin embedded sections from the central and peripheral nervous system of all three cases, and of the viscera of case 1 were studied. The sections were stained with haematoxylin and eosin ( $\mathrm{H}$ and $\mathrm{E}$ ), haematoxylin-van Giesen, periodic acid Schiff (PAS), Luxol Fast Blue (LFB), and Heidenhain's iron haematoxylin method for myelin. Frozen sections from selected tissues were stained with periodic acid Schiff, Luxol Fast Blue, Sudan black B, and Oil red O. Davenport's and Palmgren's silver impregnation methods were used to demonstrate axons. Formalin fixed material from the kidney and myocardium of case 1 was processed for electronmicroscopy. After post-fixation with $1 \%$ osmium tetroxide, the tissue was stained with saturated uranyl acetate in $70 \%$ alcohol and embedded in epon. Sections were cut at $1 \mu$ and stained with toluidine blue. 


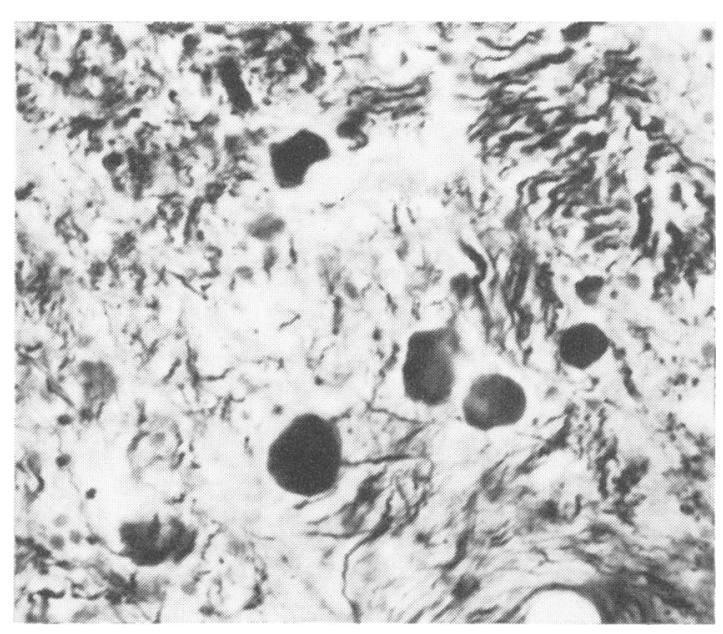

FIG. 10. Spinal cord-dorsal root entry zone. Several 'retraction balls' on degenerating axonal processes. Davenport, $\times 480$.

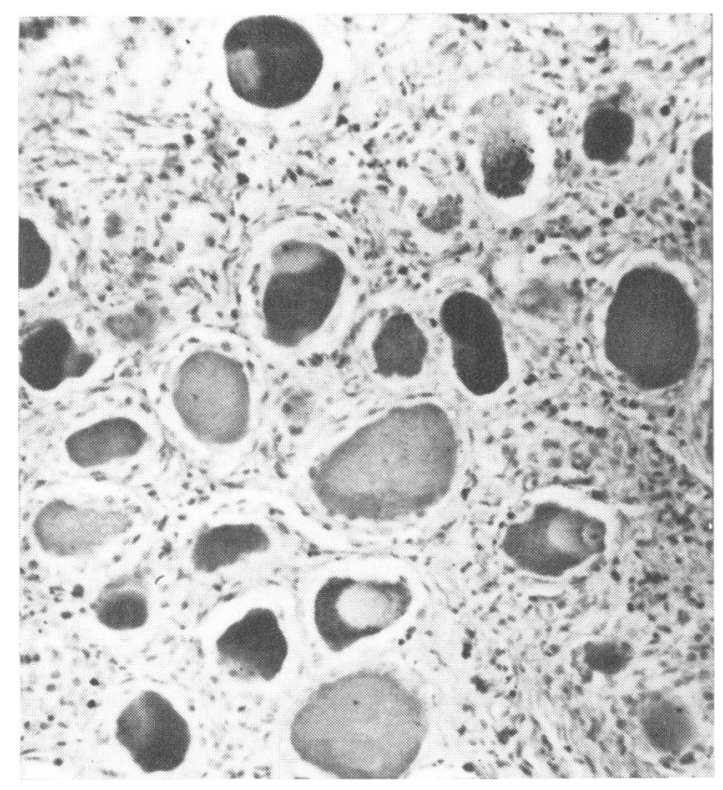

FIG. 11. Dorsal root ganglion cells-some contain abnormal glycolipid whilst others appear large and pale. Luxol fast blue, $\times 200$.
GeNerAL HISTOLOGY (Case 1) The skin lesions showed dilated vascular channels with a thin endothelial lining in the upper dermis (Fig. 2); in some instances associated with atrophy of the epidermis. In almost every tissue there was marked thickening of the media of small arteries and arterioles caused by deposition of the glycolipid (Fig. 3). This was seen in vessels of the myocardium, liver, spleen, pancreas, lymph nodes, kidney, testis, gut, thyroid, and adrenals. The substance was also seen in the form of fine granular material in foam cells in walls of vessels and in macrophages situated in interstitial tissue near the sites of accumulation. The substance was identified by its staining properties. In frozen sections it stained with neutral fat stains (Sudan black B and Oil red $O$ ) and a strong staining reaction was obtained with periodic acid Schiff and Luxol Fast Blue. In paraffin-wax embedded sections the lipid could be stained with iron haematoxylin and Luxol Fast Blue, since it is chemically similar to sphyngomyelin.

Many tubules and glomeruli in the kidney were atrophied, while others contained lipid: in tubular lining cells and in glomerular epithelial cells which showed foamy vacuolation characteristic of this condition. In some glomeruli there were large dense hyaline masses in the region of the proximal arteriole (Fig. 4). Fine and coarse masses of intracytoplasmic lipid were demonstrated in the glomerular podocytes in the epon embedded, toluidine blue stained sections (Fig. 5). There was considerable thickening of the basement membrane.

The myocardium showed swelling of the muscle fibres which had large central vacuoles. An occasional small rim of sudanophilic material was seen on the periphery of a vacuole on frozen sections but generally the vacuoles appeared empty on all frozen and paraffin-wax sections stained by all the methods mentioned. In the epon embedded, toluidine blue stained sections coarse lipid deposits were found in the vacuoles (Fig. 6). These were also seen on electronmicroscopy.

HISTOPATHOLOGY OF NERVOUS SYSTEM OF THREE CASES In the leptomeninges of the brain-stem and spinal cord there were deposits of glycolipid in the media of small arteries and arterioles. This could not be found in the leptomeningeal vessels of the cerebral hemispheres. The cerebrum was similar in all cases. Intracerebral vessels were not affected. There was no storage in the cortex and there was no abnormality in the basal ganglia or thalamus. In case 1 , serial sections of the thalamus showed no accumulation of lipid or other abnormality. In the brain of this patient there were areas of old infarction with Jense gliosis in the cerebellar cortex. The large infarct in the basis pontis was partly cystic and partly replaced by 
heavy gliosis and fibrosis (Fig. 7). The left pyramid was reduced in size and pale on sections stained for myelin: pyramidal tract degeneration secondary to the infarct in the pons.

The neurones of the supraoptic and paraventricular nuclei of the hypothalamus and those of the basolateral groups of the amygdaloid nuclei were distended by fine granules of glycolipid (Fig. 8). Several brain-stem nuclei showed neuronal storage of the abnormal lipid-the substantia nigra pigmentosa, the pontine reticular formation, the dorsal efferent nucleus of the vagus, and the salivary nuclei. In case 2 glycolipid accumulation in cells of the mesencephalic nucleus of the 5th nerve and of the nucleus ambiguus was also seen.

Sections of the spinal cord showed similar pathology at all levels in the three cases. The neurones of the intermediolateral columns contained masses of fine granules of glycolipid and numerous cells in the anterior horns in case 3 were similarly affected. In the nerve roots an occasional clump of amorphous material with the characteristic staining properties of the glycolipid was seen in the perineurium and in the medial smooth muscle of the vasa nervorum. These vessels were otherwise normal. In the dorsal root ganglia in all cases there were large, widely distributed deposits of the stored material, in the form of fine granules which distended many of the dorsal root ganglion cells and which were also seen in interstitial macrophages. Other dorsal root ganglion cells appeared large and pale and many appeared to lack nuclei (Fig. 9). Proliferation of satellite cells and residual nodules of Nageotte were prominent features. There was no myelin pallor in the posterior columns of the spinal cords. In case 1 there was pallor of the lateral columns, more severe on the right side (Fig. 10). In all cases there were groups of degenerating axons with fragmentation and 'retraction balls' in the substantia gelatinosa and in the thin, mainly unmyelinated fibres of the posterior root entry zone (Fig. 11). In cases 1 and 2 the myenteric plexuses were examined and ganglion cell storage was demonstrated. In case 3 the sympathetic ganglia of the vertebral chain were examined and did not show the neuronal storage which has been recorded by other observers (Rahman and Lindenberg, 1963). They showed, however, gross and widely distributed telangiectases resembling the angiectatic spaces in the skin (Fig. 12).

Sites of accumulation of glycolipid in nerve cells are indicated in the Table.

\section{DISCUSSION}

The distribution of the lesions in the nervous system in these three cases is similar to that

TABLE

SITES OF STORAGE IN NERVE CELLS

A. Cerebral hemispheres

1. Amygdala (basolateral nuclei)

2. Hypothalamus (supraoptic and paraventricular nuclei)

B. Brain-stem

3. Substantia nigra

4. Pontine reticular formation

5. Dorsal efferent nucleus of vagus

6. Salivary nuclei

7. Nucleus ambiguus

8. Mesencephalic nucleus of the 5th nerve

C. Spinal cord and peripheral nervous system

9. Intermediolateral columns

10. Anterior horns

11. Dorsal root ganglia

12. Auerbach's and Meissner's plexuses

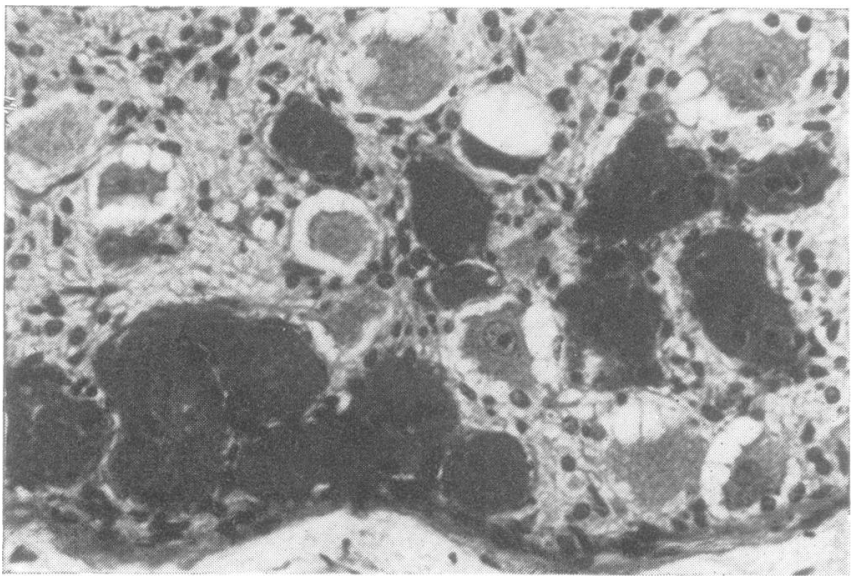

FIG. 12. Sympathetic ganglion. There are numerous large vascular spaces with endothelial lining. There is no abnormal lipid in the ganglion cells. $H$ and $E, \times 200$. 
described in previously recorded cases in many respects (Rahman and Lindenberg, 1963; Steward and Hitchcock, 1968). Previously unrecorded are the telangiectasis in vessels of sympathetic ganglia, accumulation of glycolipid in pigmented cells of the substantia nigra and in anterior horn cells of the spinal cord; and degeneration of nerve fibres in the substantia gelatinosa and dorsal root entry zone of the spinal cord.

The neuropathological interest in AndersonFabry disease centres around two featuresselectivity of neuronal involvement and the cause of the intractable pain. The selective nature of the storage phenomenon is in accordance with the other disorders of the nervous system where accumulation of substances in nerve cells results from a metabolic disorder, as, for example, in metachromatic leucodystrophy or the gangliosidoses. The selectivity of the disease process suggests that the affected neurones share a common biochemical pathway and a similar enzyme defect. The cerebral cortex, basal ganglia, and thalamus, on chemical and histological evidence are apparently not affected in this disease. Cells of the autonomic nervous system in the brain-stem, sympathetic ganglia, intermediolateral column of the spinal cord, and submucous plexuses in the gut are sites of lipid accumulation. Other sites of selective storage in the central nervous system are the amygdaloid nuclei and the anterior hypothalamic nuclei. The hypothalamic pathology is appropriate to the known clinical phenomena such as anhydrosis, intolerance to temperature extremes, a tendency to bouts of unexplained pyrexia, and diabetes insipidus which frequently contribute to the complex clinical picture. The diabetes insipidus is pitressin refractory and is most likely due to the combined effect of hypothalamic and renal tubular damage.

The unusual character of the pain in AndersonFabry disease has led to much speculation concerning its origin. Suggestions of a thalamic origin are not substantiated by the clinical features-sensory testing does not reveal a raised pain threshold; nor can any histological abnormalities be demonstrated in the thalamus. The suggestion that the pain might result from ischaemia due to involvement of vasa nervorum may be ruled out. Neither in the present study, nor in other recorded cases, has narrowing or occlusion of vasa nervorum been found. Light and electronmicroscope observations made on sural nerve biopsies in patients with AndersonFabry disease by Kocen and Thomas (1970) showed fine lipid droplets in the walls of the epineurial arterioles but otherwise normal vasa nervorum. These workers also demonstrated that there was a definite depletion of myelinated axons in the sural nerve without any evidence of segmental demyelination. The fibre loss appeared particularly to have affected the smaller nerve fibres. They point out that the fibre loss could be the result of axonal interruption at a higher level or due to loss of dorsal root ganglion cells. Bischoff et al. (1970) described similar findings in a sural nerve biopsy in which they observed degenerative changes in unmyelinated nerve fibres, the large myelinated fibres being unaffected. In ischaemic peripheral neuropathy secondary to occlusion of vasa nervorum, as for example in polyarteritis nodosa, the large myelinated fibres in the peripheral nerves are affected and cause the syndrome of mononeuritis multiplex. This is not found in Anderson-Fabry disease.

Pathological processes affecting the dorsal roots may give rise to irritative symptoms, but the occasional deposit of glycolipid in the perineurium of the dorsal roots is an unlikely explanation for the extensive bizarre sensory phenomena which are almost always present and which were prominent in the three cases discussed in this report. In these cases there was a very severe degeneration in many dorsal root ganglion cells and degeneration of small fibres in the dorsal root entry zone and the substantia gelatinosa of the spinal cord. It is the small mainly unmyelinated fibres which conduct pain stimuli. In all three cases the dorsal columns of the spinal cord which conduct the large myelinated fibres to the cuneate and gracile nuclei were unaffected. This selective involvement of small fibres is in accordance with the observations recorded on the sural nerve biopsies.

In the symptomatology of Anderson-Fabry disease the various clinical phenomena can be explained on the basis of the histopathological finding of glycolipid accumulation in vessels and tissues of various organs-for example, renal failure is due to extensive involvement of the kidney parenchyma and vessels. The phenomenon 
of pain is one feature which has not been satisfactorily explained. Pathological involvement of the dorsal root ganglion cells and the associated degenerative changes in the afferent nerve fibres in the spinal cord which subserve pain are proposed as a likely cause of the painful sensory phenomena.

I am particularly indebted to Dr. H. J. Wallace and Dr. J. Tighe for providing clinical details and postmortem material from case 3. Dr. Sabina Strich, Professor P. M. Daniel, and Dr. Ivan Janota made helpful suggestions. I should like to acknowledge the technical and photographic assistance which I received from members of the staff of the Maida Vale and the Maudsley hospitals.

\section{REFERENCES}

Anderson, W. (1898). A case of 'angeio-keratoma'. British Journal of Dermatology, 10, 113-117.

Bischoff, A., Fierz, U., Regli, F., and Ulrich, J. (1970). Peripher-neurologische klinisch-elektronenmikroskopische Befunde bei einem Fall. Klinische Wochenschrift, pp. 666671. Springer-Verlag: Berlin.

Brady, R. O., Gal, A. E., Bradley, R. M., Martensson, E., Warshaw, A. L., and Laster, L. (1967). Enzymatic defect in Fabry's disease. Ceramidetrihexosidase deficiency. New England Journal of Medicine, 276, 1163-1167.

Colombi, A., Kostyal, A., Bracher, R., Gloor, F., Mazzi, R., and Thölen, H. (1967). Angiokeratoma corporis diffusumFabry's disease. Helvetica Medica Acta, 34, 67-83.

Cumings, J. N. (1971). Inborn errors of metabolism in neurology (Wilson's disease, Refsum's disease and lipi- doses). Proceedings of the Royal Society of Medicine, 64, 313-322.

Fabry, J. (1898). Ein Beitrag zur Kenntniss der Purpura haemorrhagica nodularis (Purpura papulosa haemorrhagica Hebrae), Archiv für Dermatologie und Syphilis, 43, 187-200.

Kocen, R. S., and Thomas, P. K. (1970). Peripheral nerve involvement in Fabry's disease. Archives of Neurology, 22, 81-88.

Opitz, J. M., Stiles, F. C., Wise, D., Race, R. R., Sanger, R., von Gemmingen, G. R., Kierland, R. R., Cross, E. G., and : Groot, de, W. P. (1965). The genetics of angiokeratoma corporis diffusum (Fabry's disease) and its linkage relations with the Xg locus. American Journal of Human Genetics, 17, 325-342.

Rahman, A. N., and Lindenberg, R. (1963). The neuro- $\bar{\omega}$ pathology of hereditary dystopic lipidosis. Archives of $\mathbb{D}$ Neurology, 9, 373-385.

Spaeth, G. L., and Frost, P. (1965). Fabry's disease. Its ڤొ ocular manifestations. Archives of Ophthalmology, 74, 760769.

Steward, V. W., and Hitchcock, C. (1968). Fabry's disease. (Angiokeratoma corporis diffusum). A report of 5 cases $\omega$ with pain in the extremities as the main symptom. Pathologica Europa, 3, 377-388.

Sweeley, C. C., and Klionsky, B. (1963). Fabry's disease: classification as a sphingolipidosis and partial characteriza- $\omega$ tion of a novel glycolipid. Journal of Biological Chemistry, ? 238, 3148-3150.

Sweeley, C. C., and Klionsky, B. (1966). Glycolipid lipidosiș $\overrightarrow{0}$ Fabry's disease. In The Metabolic Basis of Inherited Diseases, pp. 618-632. Edited by J. B. Stanbury, J. B. Wyngaarden, and D. S. Fredrickson. 2nd edn. McGra年 Hill: New York.

Wallace, H. J. (1958). Angiokeratoma corporis diffusure British Journal of Dermatology, 70, 354-360.

Wallace, H. J. (1973). Anderson-Fabry disease. Briti@ Journal of Dermatology, 88, 1-23.

Wise, D., Wallace, H. J., and Jellinek, E. H. (1962) Angiokeratoma corporis diffusum. A clinical study of eigf affected families. Quarterly Journal of Medicine, 31, 17 206. 